\title{
Verschlechtert eine Angsterkrankung die Prognose nach dem Herzinfarkt?
}

\begin{abstract}
In vielen Studien konnte gezeigt werden, dass die Depression für die Entstehung und die Prognose von kardiovaskulären Erkrankungen bedeutsam ist. Wenige Studien dagegen befassen sich mit dem Zusammenhang von kardiovaskulären Erkrankungen und Angsterkrankungen. Insbesondere fehlt es an Studien, in denen die Diagnose einer generalisierten Angststörung mit Hilfe eines diagnostischen Interviews und nicht nur anhand eines Fragebogens erfasst wurde.
\end{abstract}

— 438 Patienten mit einem akuten Myokardinfarkt wurden zwischen 1997 und 2000 an vier niederländischen Krankenhäusern in die Studie eingeschlossen. Drei Monate nach dem Herzinfarkt wurde die Diagnose einer generalisierten Angststörung nach ICD-10-Diagnosekriterien anhand des "Composite International Diagnostic Interview“" (CIDI) erfasst. Zusätzlich wurde anhand des CIDI auch das Vorliegen einer Depression geprüft. Zudem wurden Alter, Geschlecht, klinische Charakteristika sowie die linksventrikuläre Ejektionsfraktion während des stationären Aufenthaltes nach dem Herzinfarkt erhoben.

Als Endpunkt der Studie wurden zum einen die Mortalität jeglicher Ursache und zum anderen ein erneuter Krankenhausaufenthalt mit einer kardiovaskulären Diagnose definiert. Die Mortalitäts- und Krankenhausaufenthaltsdaten wurden über das niederländische Zentralamt für Statistik über einen 10-Jahres-Beobachtungszeitraum bis Ende 2007 erfasst.

Anhand einer Cox-Regressions-Analyse wurde berechnet, ob sich ein Unterschied in der Überlebenszeit ohne erneute kardiovaskuläre Ereignisse zwischen den Patienten mit einer generalisierten Angststörung und den Patienten ohne eine generalisierte Angststörung nach Herzinfarkt zeigte. Die Analysen

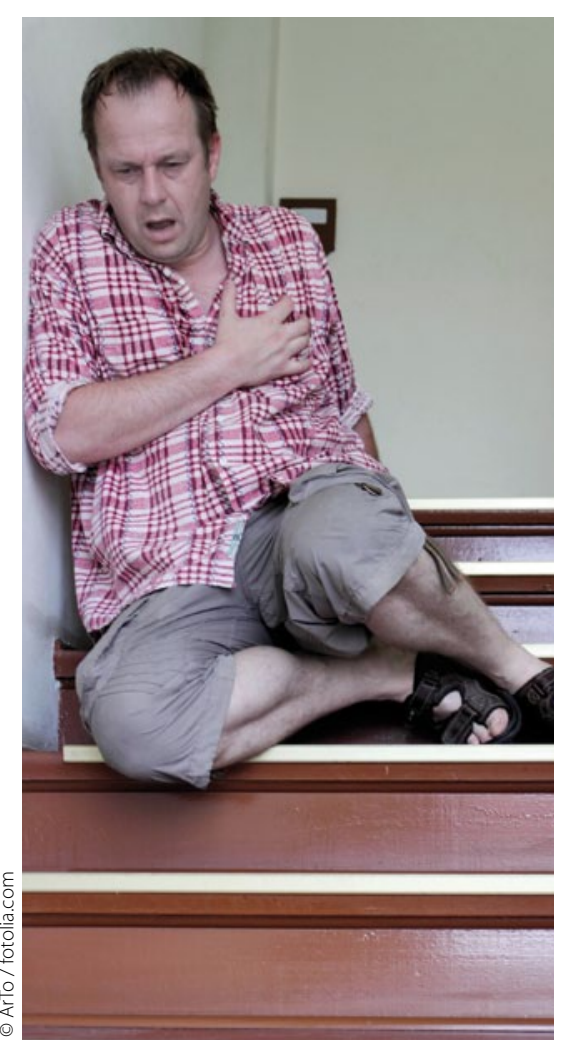

Herzinfarkt und generalisierte Angststörung: eine fatale Kombination. erfolgten unter Berücksichtigung von Alter, Geschlecht sowie der klinischen Variablen.

Von den eingeschlossenen Patienten erfüllten zum Zeitpunkt des Interviews 24 (5,5\%) die Kriterien einer generalisierten Angststörung. Insgesamt kam es in dem Beobachtungszeitraum bei 198 Patienten zu einem als Endpunkt definiertem Ereignis. Dies betraf 15 Patienten $(62,5 \%)$ mit einer generalisierten Angststörung im Vergleich zu 183 Patienten $(44,2 \%)$ ohne eine generalisierte Angsterkrankung. Unter Berücksichtigung von Alter und Geschlecht war die Diagnose einer generalisierten Angststörung mit einem erhöhten Risiko eines unerwünschten Ereignisses assoziiert (Hazard Ratio [HR]: 1,94; 95\%-Konfidenzintervall [KI]: 1,14-3,30; $\mathrm{p}=0,01$ ). Diese Assoziation zeigte sich auch nach weiteren Analysen unter Berücksichtigung der linksventrikulären Ejektionsfraktion, der Diagnose einer Depression zum Zeitpunkt nach dem Herzinfarkt und der Vorgeschichte eines Myokardinfarktes.

Kommentar

Bei Patienten mit einer generalisierten Angststörung nach einem akuten Myokardinfarkt zeigte sich in dieser Studie ein fast zweifach erhöhtes Risiko für ein unerwünschtes Ereignis (Mortalität und Krankenhausaufenthalt mit kardiovaskulärem Ereignis). Es handelt sich um die erste Studie, in der die prognostische Bedeutung der generalisierten Angststörung nach einem akuten Myokardinfarkt über einen 10-Jahres-Zeitraum untersucht wurde. Positiv hervorzuheben sind insbesondere der lange Beobachtungszeitraum und die Diagnosestellung der generalisierten Angststörung mithilfe eines standarisierten Interviews. Insgesamt ist das Evidenzniveau für eine Assoziation beider Erkrankungen aber noch als niedrig zu beurteilen. Problematisch an der vorliegenden Studie ist die geringe
Fallzahl mit lediglich 24 Patienten mit einer generalisierten Angststörung. Limitierend ist darüber hinaus, dass keine Informationen über die Dauer, den Verlauf und die Therapie der generalisierten Angststörung vorliegen. Trotz dieser Einschränkungen gibt die Studie erste Hinweise, dass neben der Depression auch die generalisierte Angststörung nach Myokardinfarkt prognostisch bedeutsam sein könnte. Dies ist von großer klinischer Relevanz. Weitere Studien sind notwendig, um diesen Zusammenhang genauer zu untersuchen.

M. NITSCH =
- PA. Roest, M. Zuidersma, P. de Jonge Myocardial infarction and generalised anxiety disorder: 10-year follow-up. Br J Psychiatry 2012; 200: 324-329 